\title{
Bio-inspired Surfaces for Fouling Resistance, A Review
}

\author{
Yichun $\mathrm{Xu}^{1, *}$, Lixinhao Yang ${ }^{2}$, and Haoran Zhang $^{3}$ \\ ${ }^{1}$ Huazhong University of Science and Technology, 1037 Luoyu Rd. Wuhan, China \\ ${ }^{2}$ Jilin University, 2699 Forward St. Changchun, China \\ ${ }^{3}$ State Key Laboratory of Marine Resource Utilization in South China Sea, Hainan University, Haikou, China
}

\begin{abstract}
Fouling causes serious problems in daily lives and mass industrial processes. Modern industry has made lots of artificial anti-fouling surfaces especially bio-inspired surfaces with some effective strategies to tackle the fouling issue. These surfaces inspired by natural creatures like lotus and sharks show both highefficiency and eco-friendly properties. This review discusses the model behind the anti-fouling properties, the mechanism of various types of fouling, and the strategy of both natural and bio-inspired surfaces. Also, the possibility of building a wide-range anti-fouling and durable surface is discussed.
\end{abstract}

\section{Introduction}

Fouling could cause serious economic and safety issues including icing of powerlines [1], coverage on airplanes [2], pipeline blockages [3], surface corrosion [4], medic instrument failure [5], extra-drag to ships [6], and other serious problems. Therefore, strategies like surface modification that can prevent or limit fouling from growing are of vital importance to reduce the negative impacts that occurred with unwanted fouling.

Fouling has different genres with different growing mechanisms. Previous researches mainly narrow in preventing a single type of fouling, while some strategies could be applied to various kinds of fouling. Among these strategies which could prevent different kinds of fouling, bio-inspired surfaces are widely discussed.

The bio-inspired design has been adapted to construct novel materials that impact a variety of fields [7]. With millions of years of natural selection, creatures have evolved peculiar structures, forms, and functions to adapt to the environment and to outweigh competitors [8]. In the aspects of fouling, many animals and plants possess distinctive body surfaces which are hydrophobic, selfcleaning, anti-adhesive, anti-corrosive, anti-fouling, antiicing, drag-reducing, self-healing, fatigue resistant, antiwearing, and so on [9]. These surfaces have inspired us to construct artificial surfaces that have similar anti-fouling structures or functions as nature ones. Moreover, almost all bio-structures are made of common elements with simple chemical structures [10], which lead us to highly efficient but easily accessible materials and devices.

This review aims to classify strategies applied by nature and related bio-inspired anti-fouling surfaces.

\subsection{Classify fouling}

Fouling is the adhesion of matters to a surface that can reduce the performance of the surface. Fouling is a broad area, including ice, clathrate, inorganic scaling, dust, paraffin and wax deposition, polymer, bio-fouling, and so on [11], which varies widely in composition and chemical structure, the scale, and modulus of different fouling distribute in a wide range.

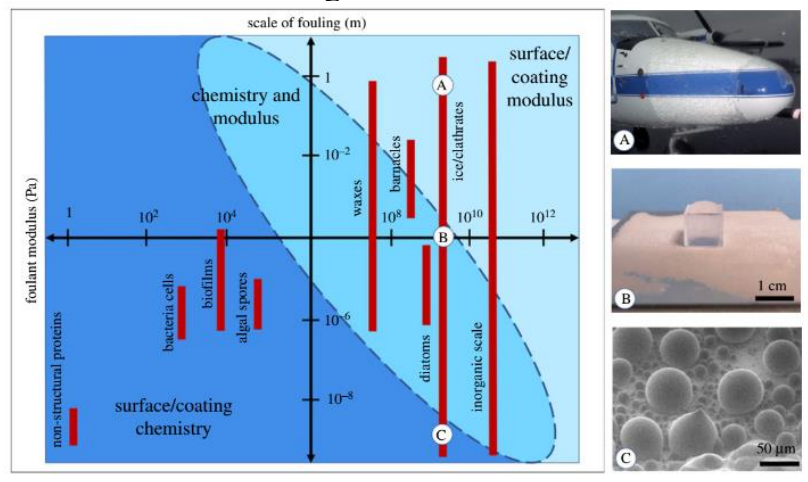

Fig. 1. Low solid adhesion surface design guide, which categorizes fouling materials according to their length scale. Images reported from [11]

The previous review divided different fouling into hard and soft fouling based on modulus and fouling scales, which is appealing and clear, shown in Fig.1. The modulus represents the adhesion energy of the fouling to a certain surface, and the scales could cover elastic strain energy and deformation of the surface [11]. But many surface construct strategies do not match this labelling. Examples include surfaces designed to prevent icenucleating or dirt attaching, using chemical adjustment strategies instead of tackling modulus of soil and ice [12]. Therefore, the classification shown in Fig. 1 is insufficient to capture the whole scope of fouling.

To make a well-organized classification, we applied the mean-shift algorithm. The mean-shift algorithm assigns data points to cluster iteratively by moving the points to the highest density data points (i.e., Clustering centroid) [13]. The centroid in the mean-shift algorithm 
could be updated in several steps until it gets the best location, the update function of the centroid is,

$$
M_{h}(x)=\frac{\sum_{i=1}^{n} G\left(\frac{x_{i}-x}{h_{i}}\right)\left(x_{i}-x\right)}{\sum_{i=1}^{n} G\left(\frac{x_{i}-x}{h_{i}}\right)}
$$

$G(x)$ is the Gaussian kernel $G(x)=e^{-\|x\|^{2}}$ which could be changed to other kernels as well. The $x$ is the temper centroid point, and $x_{i}$ is other points. $h_{i}$ refers to the normalized constant [14].

Fouling data is randomly generated in reported possible zones and then get classified by the algorithm. As only modulus and scale data are fed into the algorithm, the result mainly shows classifications based on the two properties.

The result shows that fouling is divided into 4 genres. Protein fouling is labelled individually as the modulus and the scale of proteins is much smaller even comparing to rather small-scale bacteria, and not similar to any other fouling. Bio- and dust-fouling are classified into one kind as they share similar modulus and fouling scales, with different bio-fouling like bacterial and algal shares similar forming processes. Inorganic fouling is divided into two genres by the algorithm, based on the scale, which also makes sense with the fouling formation mechanism varies on different scales. Therefore, the classification that divided fouling into protein fouling, bio- and dust-fouling, micro-inorganic fouling, and macro-inorganic fouling is reasonable.

It should be mentioned that the barnacles are divided into macro-inorganic fouling in the chart, which indicates that the mechanisms behind this type of fouling maybe not so bio-based, the progress of barnacle growing might contains some inorganic steps.

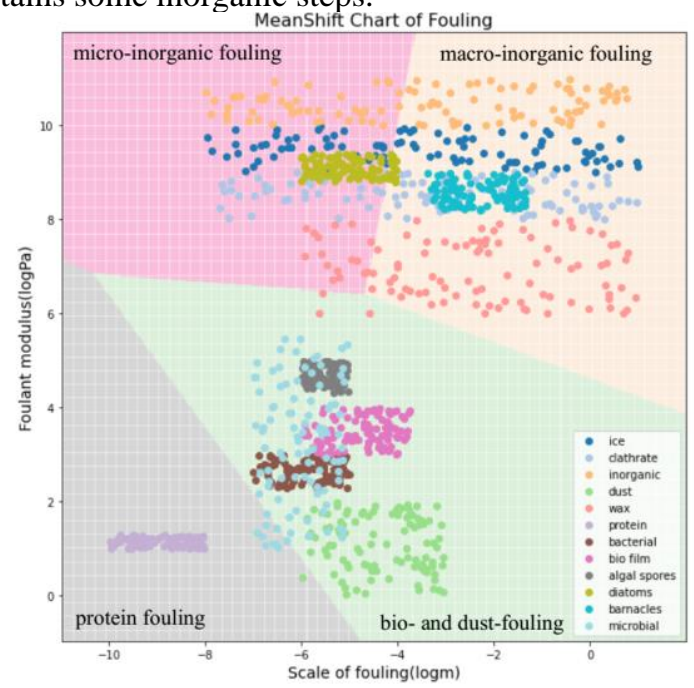

Fig. 2. Mean-shift Chart of different fouling. Which categorizes fouling materials according to their length scale and modulus.

\subsection{Bio-inspired strategies to make anti-fouling surface}

The strategies to build functional fouling resistant surfaces include nano- or micro-structures [15], chemical treatments [16], and other strategies like SLIPS [17].
Changing surface micro-structure which operates well in liquid fouling is a fundamental strategy to construct anti-fouling surfaces especially fouling with low modulus like dust [18]. While in cases of high modulus fouling, micro-texture is typically detrimental as it increases the adhesive contacting surfaces for an interlocking interface across which de-bonding is significantly hindered [19]. It should also be mentioned that the degree of fouling resistance has little relationship with the hydrophobicity of the surface. Lotus leaves like superhydrophobic surfaces have shown great self-clean properties to dust fouling and other low adhesion fouling [33]. Superhydrophilic shark skin-inspired artificial surfaces have also contributed a lot to low drag and less attachment of bio- or marine-fouling. However, facing some kinds of fouling like ice fouling, the superhydrophobic or superhydrophilic structures cast a negative impact on the fouling resistant properties [21].

The chemical treatment strategy aims mainly to lower the substrate surface energy, reduce the bond between the surface and the fouling, known as the work of adhesion, $W_{a}$. The contribution of the interfacial free energy of a surface $\left(\gamma_{s 1 v}\right)$ to the work of adhesion is commonly represented by the equation $W_{a}=\gamma_{s 1 v}+$ $\gamma_{s 2 v}-\gamma_{s 1 s 2}$, where $\gamma_{s 1 v}$ and $\gamma_{s 1 s 2}$ are the interfacial free energy for the foulant and the foulant-substrate interface. Nature surfaces often apply low surface energy materials like wax, and silicones or fluorinated polymers are often applied to artificial surfaces based on the strategy. Also, surface chemistries that interact specifically with a fouling material could be quite functional, researches have been conducted in enzymes [22], AFPs (antifreezing protein) [23] and other fields.

The SLIPS (Slippery Liquid-Infused Porous Surfaces) structure uses liquid-containing porous or textured structures to create a slippery surface that moves fouling away [11]. SLIPS present not only non-wetting property to most liquid but also anti-biofouling performance to the bacteria and other fouling like ice or oil [24]. The mechanism of SLIPS is discussed in the chapter below, in all, SLIPS can function well in various fouling types.

\section{Lotus leaf inspired surfaces}

\subsection{Natural lotus leaves}

Lotus leaf is one of the most well-known superhydrophobic surfaces. It is believed that since lotus plants thrive in humid, marshy environments, which cast more probability of bio-fouling or dust attachment, the self-cleaning property of the surface is needed to guarantee photosynthesis.

In nature, rain droplets impact the lotus leaf surface and roll-off due to the surface's water repellent property. This leads to self-cleaning, where droplets collect and remove the contaminant particles. The ability of lotus-like surfaces for self-cleaning and water-resistant was known as the lotus effect. The lotus effect relies on roughnessinduced superhydrophobicity and low adhesion, where air pockets are presented at the solid-liquid interface [25]. In 
conclusion, the superhydrophobic lotus surface could prevent low modulus fouling like dust.
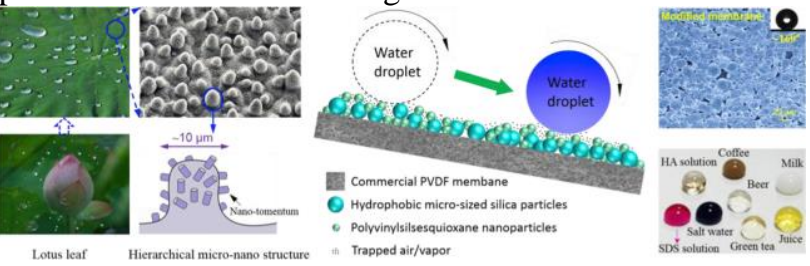

Fig. 3. Artificial superhydrophobic hierarchical micro-nano scale rough surface structures and original lotus leaf structure. Images reported from [26].

Fully developed lotus leaves result in a nearly spherical water droplet on the surface. As shown in Fig, the surface contains hierarchical structures composed of micro-sized protrusions and nano-sized wax tubules. By combining the hierarchical structures with the lowsurface-energy wax, the lotus surface gains superhydrophobicity [18]. It should be mentioned that some other fauna and flora species also exhibit lotus-like self-cleaning properties [27]. Their surfaces contain superhydrophobic structures which result in self-cleaning properties.

Recent findings have also shown that lotus surfaces have present surprisingly excellent bio-fouling resistant features, ascribing to the stable air cushion entrapped at liquid/solid interfaces, which significantly reduces the contacting area and minimizes the biofouling [28]. Moreover, the lotus surface shows an inherent bactericidal property. In previous work, researchers have shown that nearly all of the cells adhered on the lotus leaf are dead in $24 \mathrm{~h}$ of time. Bacterial inactivation on the lotus leaf surface originates from the mechanical rupture property of the cells, which is obtained by densely assembled nanotubes with a high aspect ratio [29]. However, bacterial proliferation and colonization progress are inevitable, since the lotus surface can't kill all bacteria [30].

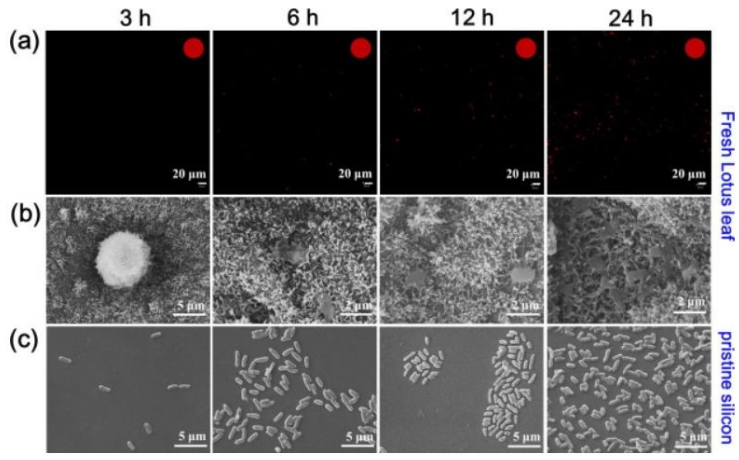

Fig. 4. Representative SEM and CLSM images of bacteria on the pristine silicon and fresh lotus leaf surfaces after $3 \mathrm{~h}, 6 \mathrm{~h}$, $12 \mathrm{~h}$, and $24 \mathrm{~h}$ incubation. Images reported from [30].

\subsection{Lotus leave inspired artificial structure}

Inspired by the lotus leaf, superhydrophobic hierarchical micro-nano scale rough surface structures are applied in artificial fouling resistant surfaces. Due to the superior anti-wetting and anti-fouling properties of the structure, the fabricated surface is promising and feasible in occasions like desalination with membrane distillation, especially for challenging saline wastewaters with strong fouling or pore wetting potential.

Previous research presents a surface constructed by synthetic silica composite particles on a commercial PVDF membrane comprising modified micro-sized silica particles (SiPs) with spherical polyvinylsilsesquioxane nanoparticles grafted onto the SiPs surfaces [26]. The resultant superhydrophobic membrane presented robust membrane distillation performance compared with the neat PVDF membrane. This indicates that this kind of surface fabricating method could result in good selfcleaning and anti-dust surfaces.

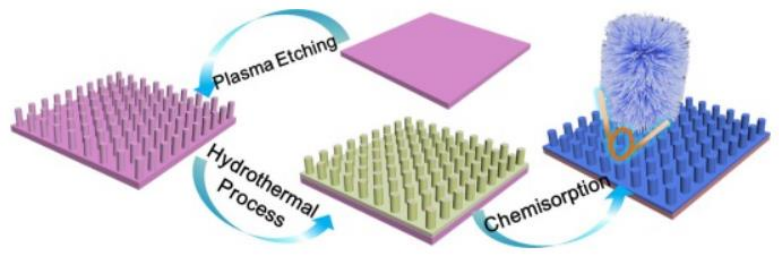

Fig. 5. Schematic illustration of fabrication procedure of lotus leaf-like structures. Images reported from [30].

As discussed in the natural surface part, the lotus leaves' nanostructure also shows anti-bio-fouling properties. Inspired by this synergistic antibacterial effect, Rujian Jianga et al. construct a hierarchical surface with both micro-scale cylinders patterned silicon wafer and nano-scale $\mathrm{ZnO}$ needles [30], shown in Fig.5. The bactericidal experiments are conducted in their research and the artificial surfaces present strong bactericidal activities that more than $98 \%$ of bactericidal efficiency can be achieved over the full incubating time of E. coli [30].

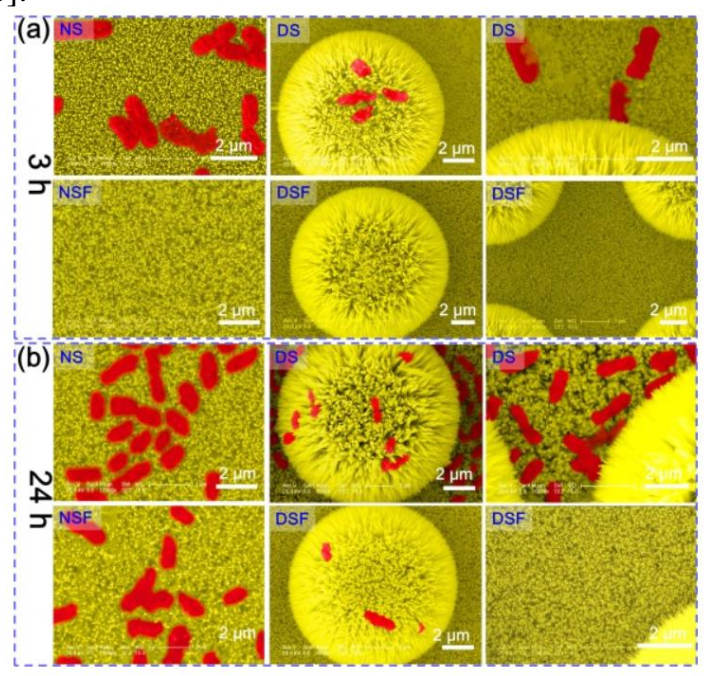

Fig. 6. Representative SEM images of $E$. coli morphologies adhered on the samples. The pictures are processed with falsecolour. Images reported from [30].

\section{Shark skin inspired surfaces}

\subsection{Shark skin effect}

Sharkskin is another well-known natural anti-fouling and self-cleaning surface. Sharks require low drag skin to move fast and catch prey, and the property of anti-fouling 
is necessary to maintain a low-drag-rate skin. Comparing to other aquatic species, shark skin is featured by highly efficient fouling resistance behavior. As a comparison, whales are often covered with barnacles, shown in Fig.7.
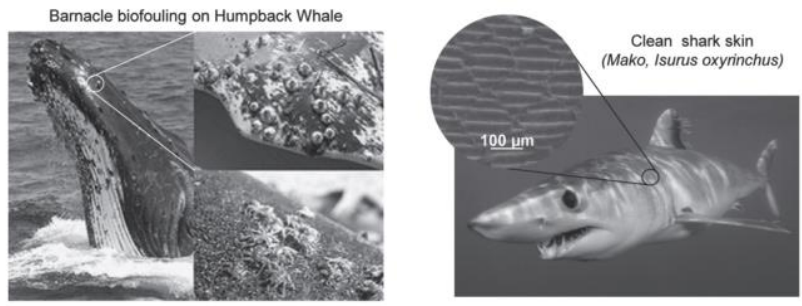

Fig. 7. Images highlight differences between Humpback whale and Mako shark. Images reported from [31].

Sharkskin is equipped with specially sized microscales called dermal denticles, which can effectively control the turbulent vortex, reducing momentum transfer and shear stress, and thus reduces drag [32]. The riblets lift and presumably pin any vortices generated in the solid-liquid interface, and could reduce the cross-stream motion of the fluid, thus contributes to low drag [33]. Lower drag increases fluid flow at the skin, reduces microorganism settlement time, promotes washing, therefore contributes to the anti-fouling property.
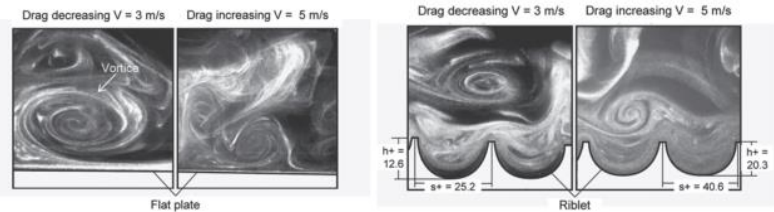

Fig. 8. Flow visualization images using smoke from atomized oil burned in air to study the turbulent vortices behaviour with and without riblets at two velocities. Images reported from [31].

In addition to low drag, it is difficult for microorganisms to adhere to and colonize shark skin with the microstructure on the skin prevents them that prefer specific trench widths and depths from settling. Also, flexion of scales and a mucous layer contributes to antifouling as well [34].

Other aquatic creatures' skin like fish scale also shows anti-biofouling properties [35], the function of fish scale is similar to sharkskin with micro-structures and hydrophilic property. However, the scales of various fish genres are often not as effective as the sharkskin.

\subsection{Artificial sharkskin-like surfaces}

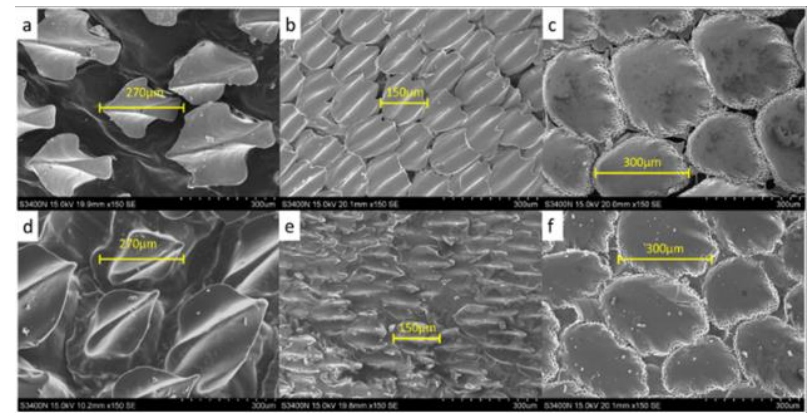

Fig. 9. SEM images of $(a-c)$ actual shark skin structures, $(d-f)$ artificial PDMS membranes. Images reported from [33].
Highly efficient shark skin effects inspire researchers to imitate the structure and the mechanism. By replicating the actual shark skin structure, Lin et al. build an artificial surface based on PDMS membrane [33]. Results of the anti-adhesion ability of the surfaces show that these sharkskin replica structures are more effective to prevent bacteria adhesion, compared with the pristine PDMS.

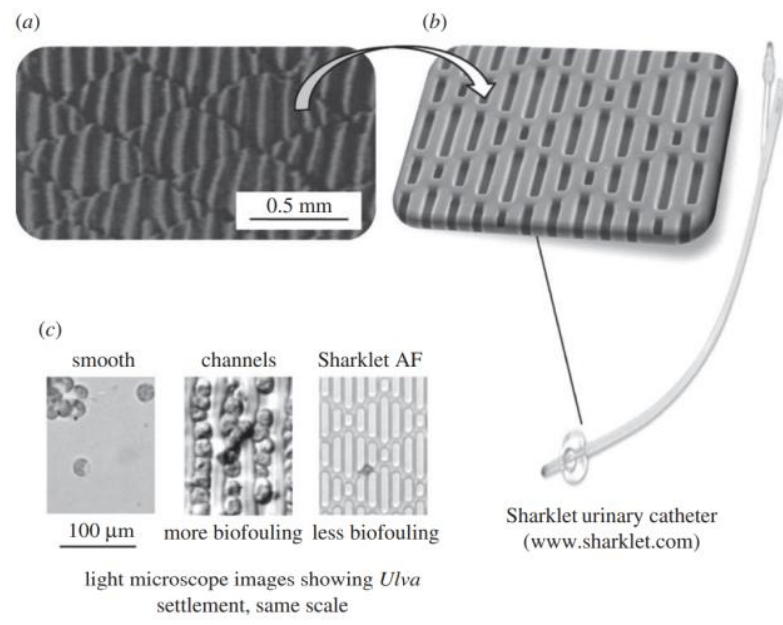

Fig. 10. Sharklet urinary catheter (b) and experiments (c) demonstrate the Sharklet antifouling effectiveness with Ulva settlement on various topographies (adapted from Carman et al. [36].). Images reported from [10].

More than just imitate the real shark skin, microtexture films with riblet-inspired antifouling properties are invented and thoroughly researched. By optimizing the blade thickness and spacing, Chien et al. found that sharkskin-inspired micro-textures can reduce drag by up to 9.9 percent [37]. Also, Sharklet $\mathrm{AF}^{\mathrm{TM}}$, a shark skininspired commercial coating, shows that the structure has better biofouling resistant ability than simple flat surfaces or channelled textures.

Other sharkskin-inspired drag-reducing products include the experimental $3 \mathrm{M}$ saw tooth riblets as well as the Speedo FastSkin fabric racing swimsuit. The 3M riblets are manufactured in polymer films and have been tested in various aerospace, marine, and industrial applications [8]. Sharkskin effect has captured the attention of the National Aeronautics and Space Administration (NASA), US Navy, Airbus, Boeing, and other industry giants. Reducing fluid drag can also benefit wind turbines, microfluidics, and oil pipeline industries in case of fouling prevention.

\section{Surfaces with anisotropic structures}

\subsection{Rice leaves and butterfly wings}

Individual natural structures like rice leaves and butterfly wings exploit an anisotropic structure. Since rice plants thrive in humid, marshy environments, self-cleaning prevents dirt or bio-fouling from attaching, which may inhibit photosynthesis [38]. Since butterflies are weak and unable to clean their wings, it is critical for maintaining structural coloration and flight control [15]. Both rice leaves and butterfly wings exhibit unique anisotropic flow characteristics [8]. The mechanisms of rice leaves and 
butterfly wings could be considered as a combination of lotus and sharkskin effect [15].

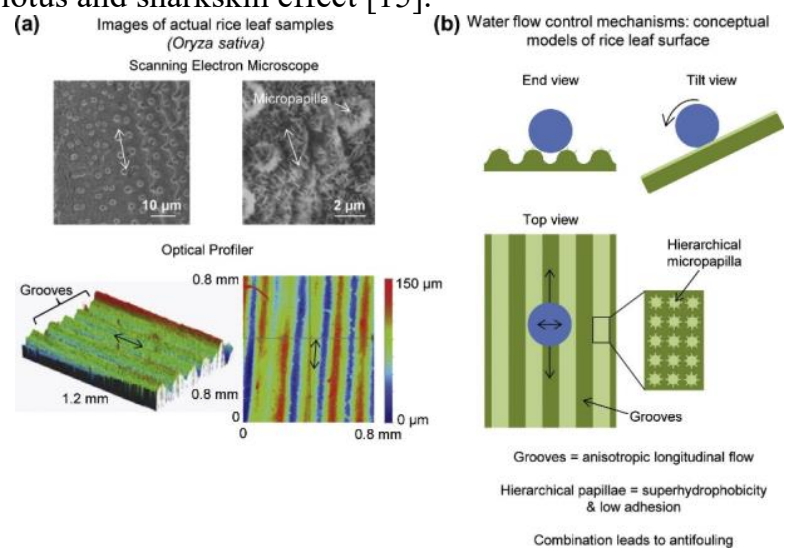

Fig. 11. Optical profiler height maps of (a) actual rice leaf as well as (b) a conceptual model. Images reported from [15].

The rice leaves are hydrophobic and have a stripe structure with individual hierarchical micropapillary, shown in Fig.11. The micro-and nano-structures are similar to those of the lotus leaf. While on top of the surface, the papillae with an average diameter of about 5$8 \mathrm{um}$ are arranged in one-dimensional order parallel to the leaf edge and randomly in the other directions. It is the special structure that provides anisotropic flow, superhydrophobicity, and low adhesion properties. The combination of anisotropic flow, superhydrophobicity, and low adhesion can facilitate self-cleaning. A simplified conceptual model of rice leaf hierarchical structures is shown in Fig.11b. The water droplets sit above the hierarchical surface structures and can effortlessly roll and collect contaminants to improve self-cleaning efficiency [38].

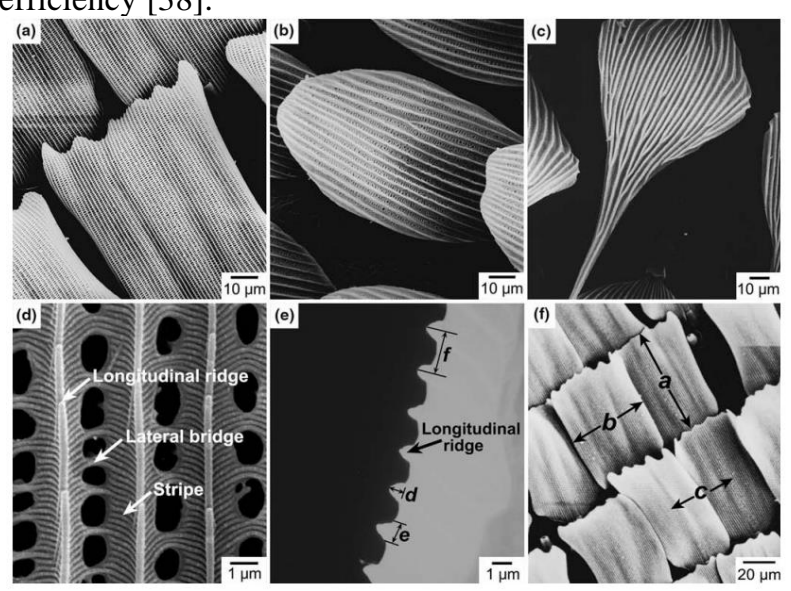

Fig. 12. Multiple-dimensional micro/nanostructures of the butterfly wing surface (SEM). Images reported from [39].

The butterfly wing surfaces have complicated microor nano-hierarchical structures. The multiple dimensional micromorphology contributes to the special complex wettability of wing surfaces [38]. The butterfly wing surfaces share some similarities with the lotus leaf like low surface energy and superhydrophobicity [28]. The butterfly wing surfaces are composed of naturally hydrophobic material (chitin, protein, fat, etc.). The spaces in the skeleton of the wing surfaces are occupied by air, therefore, water droplets stand at the tips of the skeleton structure [15]. There are significant differences in various directions of the wing. When the water droplet slides in a direction parallel to the longitude direction, the spaces between the scales are filled up by air. Both the contact area and the adhesive force between the droplet and the wing surface are fairly small. Even a very slight tilting of the wing is sufficient to cause the water droplet to roll off readily and take away the contaminating particles efficiently. This marvellous property endows butterfly wings the ability of directional self-cleaning in a watery environment [39].

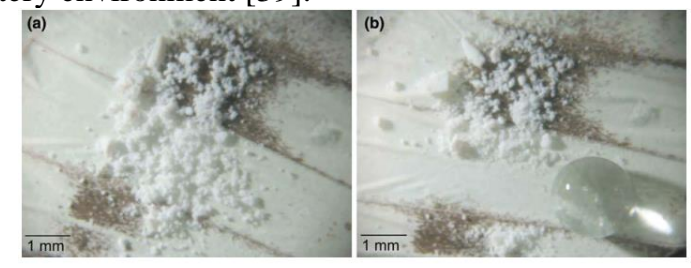

Fig. 13. Removal of contaminating $\mathrm{CaCO}_{3}$ particles from the butterfly (Pontia daplidice) wing surface by a rolling water droplet. Images reported from [39].

\subsection{Artificial anisotropic geometries}

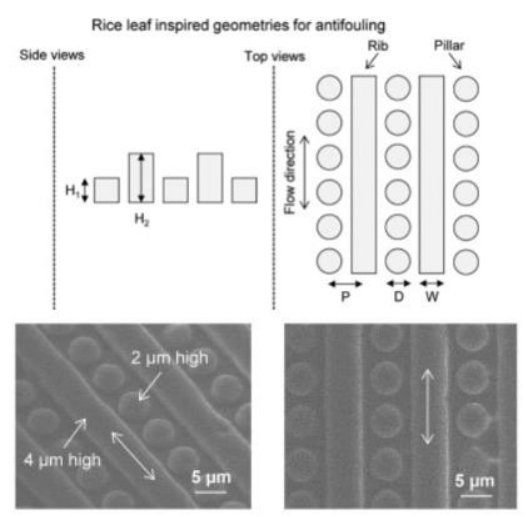

Fig. 14. Rice leaf-inspired geometries for antifouling. Images reported from [15].

The structure of rice leaves and butterfly wings inspire the design of artificial surfaces. Gregory Bixler et al. reported a rice leaf-inspired surface with great antifouling benefit, the structure of the surface is shown in fig.14. An incubation test shows that the surface could reduce coverage of biofouling by up to $33 \%$. Also, a series of self-cleaning wash experiments indicates that the artificial surface could reduce $86 \%$ of contaminant [38]. This indicates that the rice leaf and butterfly wing effect may be designed using a uniform micropattern of superhydrophobic low adhesion cylindrical pillars arranged in longitudinal rows.

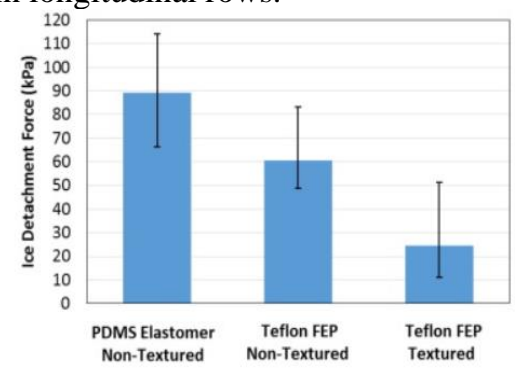

Fig. 15. Ice adhesion strength of the textured superhydrophobic FEP sheet compared to non-textured FEP sheet and nontextured PDMS elastomer film. Images reported from [40]. 
The artificial surfaces' anti-fouling performance could be enhanced by combining chemical treatment with micro-texture. Yeong et al. found that the ice adhesion strength of the superhydrophobic sheet could be reduced by adding rice-leaf-like textures. As shown in Fig.15, the film they made with Teflon FEP and texture has a $20 \mathrm{kPa}$ on average ice detachment force, which is significantly lower than non-textured surfaces [40]. This indicates that micro-texture could help reduce ice fouling combining with other surface constructing strategies.

\section{Re-entrant surfaces}

\subsection{Micro-hoodoo surfaces}

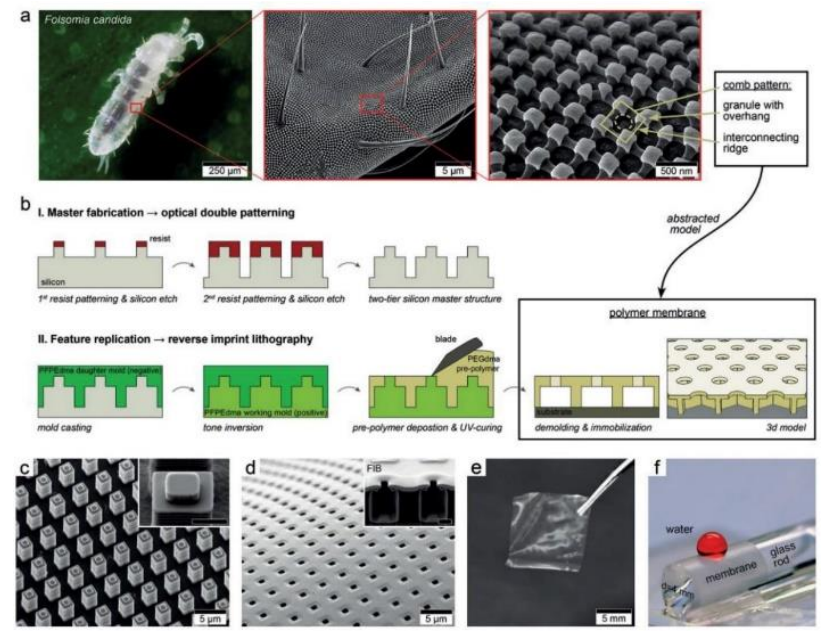

Fig. 16. Springtail skin morphology and process scheme for manufacturing polymer membranes with similar structural features. Images reported from [41].

The micro-hoodoo structure is inspired by springtail surfaces that show omniphobicity, which could contribute to a self-cleaning and anti-oil-fouling surface. The surfaces of the springtail are so omniphobic that it will still be covered with a layer of air even be drunk in water [42].

Inspired by the fascinating discoveries, Hensel et al. design delicate re-entrant morphologies with a high resolution of a few tens of nanometres. The skin structure of Tetrodontophora bielanensis (a European giant springtail) was translated into polymer replicas [41], shown in Fig.16. Hensel et al. have also successfully fabricated an omniphobic membrane via multi-step reverse imprint lithography [43]. The resultant membrane exhibited great omniphobic properties and could reduce attaching of various fouling like dust and crude oil.

\subsection{Micro-mushroom surfaces}

Another surface with a special texture that shows great similarity to the "micro-hoodoo" structure was developed by Tan et al. who referred to the texture as "micromushrooms" [44]. To test the performance of the modified membrane, they placed it into an aqueous precursor solution. Due to the superhydrophobic nature of the membrane, it remained afloat on the solution surface, and the precursor solution would not contact the microneedles except at their tips. The resultant surface showed omniphobicity as reflected by a remarkable contact angle of $100^{\circ}$ with n-hexadecane.

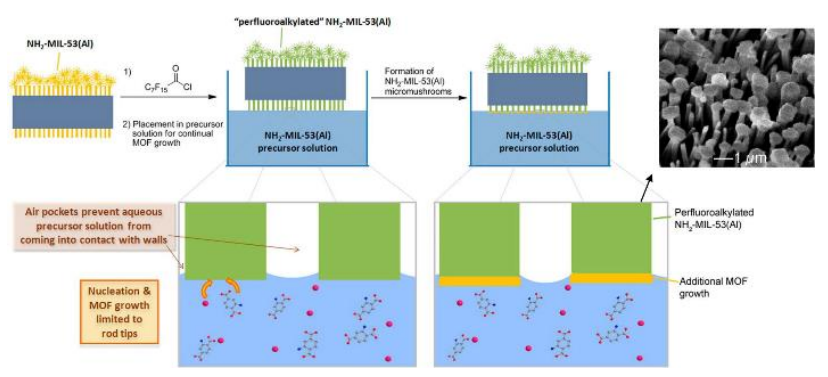

Fig. 17. Graphical representation of NH2-MIL-53(Al) micromushroom synthesis through interfacial continual growth. Images reported from [44].

Both micro-hoodoo or micro-mushroom structure could lead us to a highly functional self-clean and anti-oilfouling surface.

\section{Bio-inspired surfaces with molecules}

\subsection{Seaweed excretions}

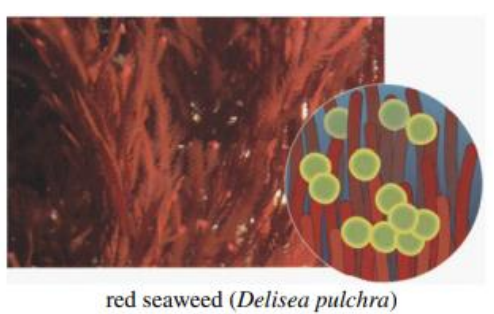

Fig. 18. Red seaweed using a halogenated furanone to manipulate colonizing bacteria 'attraction' messages. Images reported from [45].

Chemical defence is another commonly used approach to prevent fouling in nature. Many creatures could excrete chemical components which have great anti-fouling mechanisms. Seaweed secretion could reduce attachments of bacteria or other fouling [46]. Kulwadee Karnjana et al. found that the ethanolic and furanone eradicated from red seaweed Gracilariafisheri could significantly diminish bacterial infections in shrimps. The excreted chemical is highly bactericidal, which could get rid of bio- or marine-fouling attachments [45]. Another red seaweed Deliseapulchra could excrete a halogenated furanone to manipulate colonizing bacteria 'attraction' messages, which could fool bacteria that the seaweed has already been colonized. Therefore, prevent the surface from potential bio-fouling attachments [47]. One other plant, the eelgrass (Zosteramarina), could produce zosteric acid. Zosteric acid is a sulphury phenolic acid, which inhibits the accumulation of bio-fouling by interfering with adhesion [8].

The bio-chemical-inspired artificial organic coatings are easily imitated. Therefore, the strategy has been widely used in occasions like ship anti-fouling paintings [11]. 


\subsection{Pilot Whale Skin}

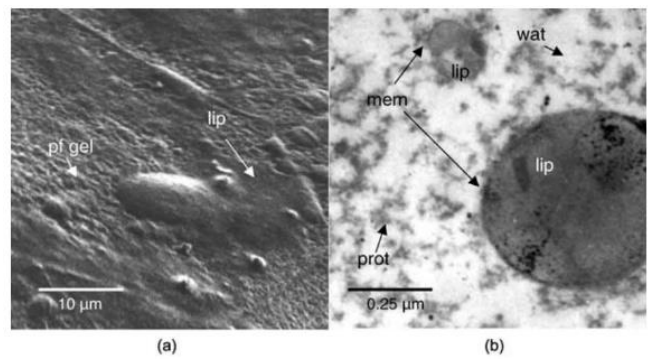

Fig. 19. CSM and TEM micrograph of the skin surface of $G$. melas. Images reported from [48].

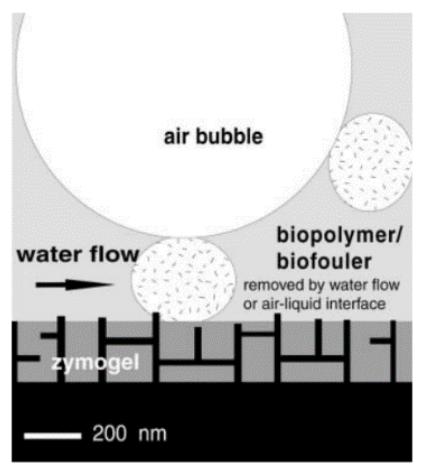

Fig. 20. Preliminary model of the self-cleaning abilities of the pilot whale (G. melas) skin. Images reported from [49].

Dolphins also have skins that could resist marine fouling like barnacles, while the mechanism is different from sharks. On the skin surface of delphinids, small biofouling is exposed to high shearwater flow and liquidvapor interfaces of air-bubbles during dolphin jumping. Previous research has shown that the mechanism is a combination of mechanical cleaning and chemical secretions [8].

Research on the pilot whale, a kind of dolphin, has shown that the state of self-cleaning is supported by the even, nano-rough gel-coated epidermal surface of the skin [48]. The skin surface contains a specialized nanostructure that could delay the attachment of microscopic organisms such as barnacle larvae.

In between the ridges is a rubber-like gel containing enzymes that denature proteins and carbohydrates [49], shown in Fig.20. The gel, which oozes out of the gaps between skin cells, is replenished as the whale sheds its skin. The gel-coat of the skin surface is a chemically heterogeneous skin product, which contributes to the ablation of traces of the biofouling process. Researchers think that organisms such as bacteria and diatoms have trouble sticking to the ridge edges, which provide little purchase. And if they try hanging onto the gel the enzymes will attack them [49].

\subsection{Synthetic surfaces inspired by enzymes}

The use of enzymes produced by various living things in anti-fouling especially anti-bio-fouling has been investigated continuously over the past 30 years [8]. Enzymes have shown superiority in fouling riding [22]. Also, the enzymes are environmentally friendly, they rapidly biodegraded once released from the coating, which shows great potential to make a useful anti-fouling surface.

The main challenge of enzyme-based anti-fouling technology is how to retain activity for a realistic or commercially viable period. In several cases, short-term anti-fouling activity in coatings has been proven, but long-term efficiency towards all fouling organisms remains to be reported. This obstacle can be ascribed to difficulties related to coating production, storage, and operation. Based on the limited number of commercial products available, simply mixing enzymes and paint is ostensibly insufficient, and therefore some sort of enzymatic stabilization and controlled release must be achieved for the concept to fulfil its potential.

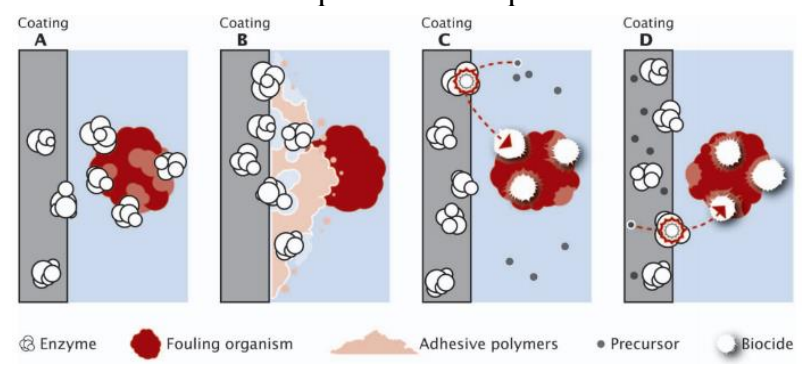

Fig. 21. Proposed mechanisms of enzymatic anti-fouling. Images reported from [22].

\subsection{AFP}

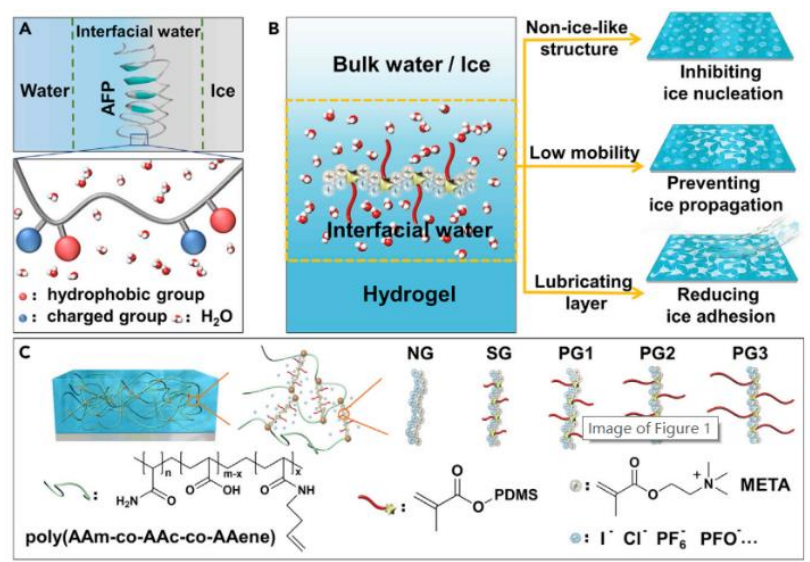

Fig. 22. Structure of AFPs and the chemical structures of the artificial surface. Images reported from [23].

In nature, some plants, insects, and fishes produce AFPs to control ice formation so that they can survive in sub-zero environments. AFPs can depress the freezing temperature and achieve a temperature difference between the freezing and melting temperatures of ice, which is termed as thermal hysteresis. Inspired by the unique functions of AFPs on shaping ice crystals and inhibiting ice growth and recrystallization, rich varieties of materials have been developed to control ice formation.

AFP's anti-freeze functions are achieved through tuning the structure, mobility, and amount of interfacial water, which respectively regulate the ice nucleation, growth, and adhesion [23]. The structure of AFP is shown in Fig.22, with hydrophobic groups and hydrogenbonding with water. Both hydrogen-bonding and hydrophobic groups contribute greatly to the regulation of 
interfacial water, thus leading to the AFPs with enhanced anti-freeze activity.

Previous researches provide several AFP-like polymers such as OCNs or OQCNs [50]. Crosslinking with some organic chains could make a more durable and functional surface. These multifunctional anti-icing surfaces can delay ice formation remarkably without altering its original ultralow ice adhesion. Compared with the multifunctional anti-icing properties, the mechanical robustness of this hydrogel coating is less prominent, which may limit its widespread application in real-life conditions. The development of an anti-icing hydrogel that has extraordinary mechanical properties such as double-network hydrogels, inorganic/hydrogel hybrids, and hydrogel fibres is still urgently needed.
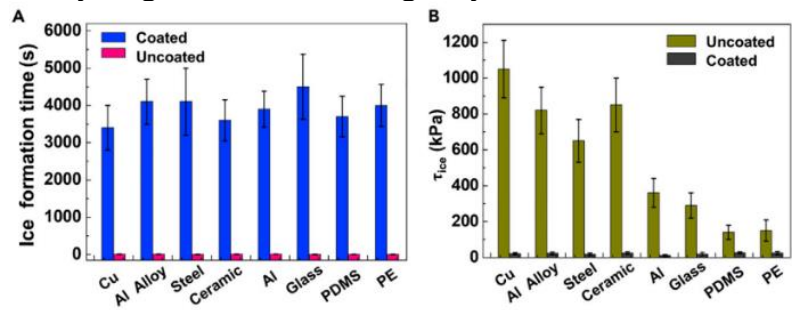

Fig. 23. Anti-icing applications on AFP coatings. Images reported from [50].

\section{SLIPS}

\subsection{Pitcher plant}

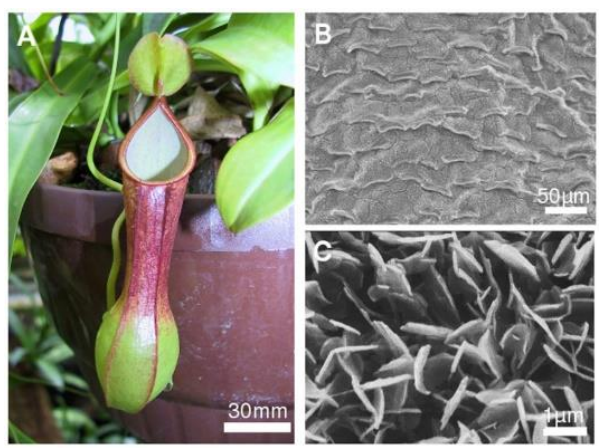

Fig. 24. Morphology of a pitcher of the carnivorous plant Nepenthesalata. Images reported from [51].

Plants of the carnivorous genus Nepenthes have highly efficient attract traps that could retain and finally digest animal prey, insects slide on its fully wettable anisotropic surface [51]. The peristome of the trap was shown to be completely wettable by nectar or rainwater, leading to coverage with homogeneous liquid films that impede close contact between the plant and tarsal pad surfaces. The surface of the conductive zone immediately below the peristome has also been recognized as essential for trapping and retaining insect prey, but in this case due to its special downward-directed lunate cells and its coverage with a thick layer of epicuticular wax crystals [52]. Despite these very different mechanisms, both surfaces are extremely slippery for insects and cause the prey to fall into the lower part of the pitcher.

The structure of the pitcher plant results in a low adhesion rate to almost all attaching solid materials, which therefore maintain its cleaning through insects and dust. Also, researchers have shown that the surface of pitcher plant has properties like anti-oil-fouling which is needless in the natural environment [53].
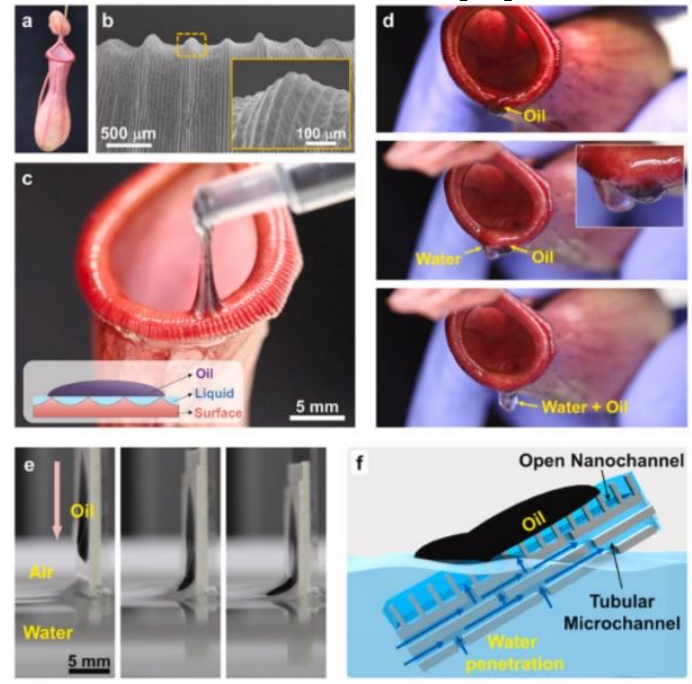

Fig. 25. Infused-liquid-induced oil removal on Nepenthesalata. Images reported from [53].

Artificial SLIPS is inspired by the pitcher plant. Like the pitcher plant surfaces, SLIPS present not only nonwetting property to most liquid [24], but also antibiofouling performance to the bacteria and other fouling like ice [54] or oil [55].

\subsection{SLIPS on biofouling}

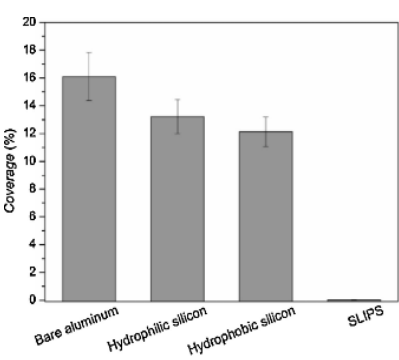

(a)

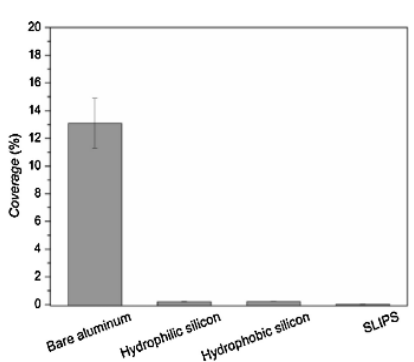

(b)
Fig. 26. The coverage of $C$. vulgaris settled on four kinds of surfaces. Images reported from [56].

Artificial SLIPS presents not only non-wetting property to most liquid but also exceptional anti-biofouling performance [56]. Wang et al. reported an anti-biofouling SLIPS which was prepared on aluminum by a three-step procedure [56]. The so-made SLIPS and control group (bare aluminum, hydrophilic silicon wafer, hydrophobic silicon wafer) are immersed in both static and dynamic culture media with $C$. vulgaris for 10 days. The result suggests that the liquid-like property of SLIPS, which can reduce the settlement of $C$. vulgaris in a static condition can also contribute to the exceptional anti-biofouling performance of SLIPS in dynamic conditions. According to this research, it can be expected that the SLIPS has a great potential in marine and other anti-biofouling applications [56].

Other SLIPSs show great anti-bio-fouling properties as well. PFPE lubricant-infused three-dimensional 
nanostructures TC4 titanium alloys could significantly reduce the adhesion of chlorella and $P$. tricornutum on titanium alloys [57]. A self-healing slippery organogel layer by infusing the silicone oil into PDMS-PUa with dynamic hydrogen bonding performed excellent antifouling performance after immersion in the culture solution inoculated with bacteria [55].

\subsection{SLIPS on ice}

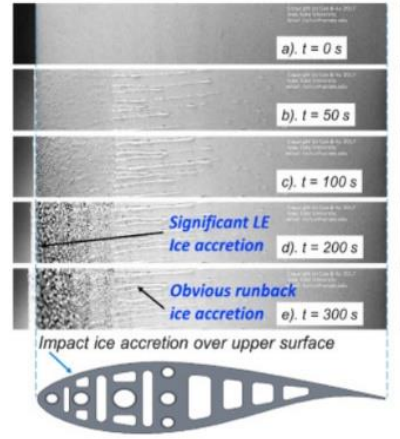

(a). Uncoated Turbine blade model

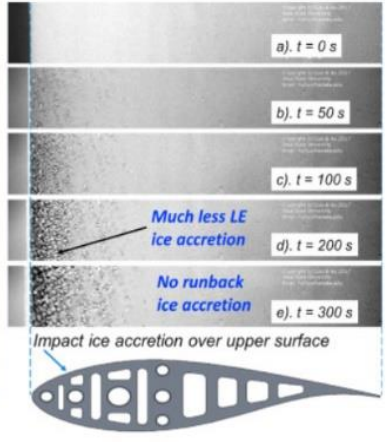

(b). Turbine blade model coated with SLIPS
Fig. 27. Typical snapshot images to reveal the dynamic ice accretion process over the surface of the turbine blade models before and after coated with SLIPS. Images reported from [24].

Ma et al. reported a SLIPS with great ice-fouling resistant property by infusing a lubricant fluid of DuPont's Krytox $^{\circledR} 103$ into a porous layer of nanofibrous PTFE membrane [24]. As shown in Fig.27b, after applying the SLIPS to coat/cover the turbine blade model, in addition to having much less ice accretion in the region near the air foil leading edge, no runback water or ice accretion could be observed on the SLIPS coated turbine surface. The result shows that there is much less ice accretion on the SLIPS coated test model. As a result, the total amount of the water mass that can be frozen into ice over the SLIPS coated blade surface would become much smaller, in compassion to the case with an uncoated test model. Therefore, much less ice accretion was found in the region near the air foil leading edge for the SLIPS coated blade model.

\subsection{SLIPS on other fouling}

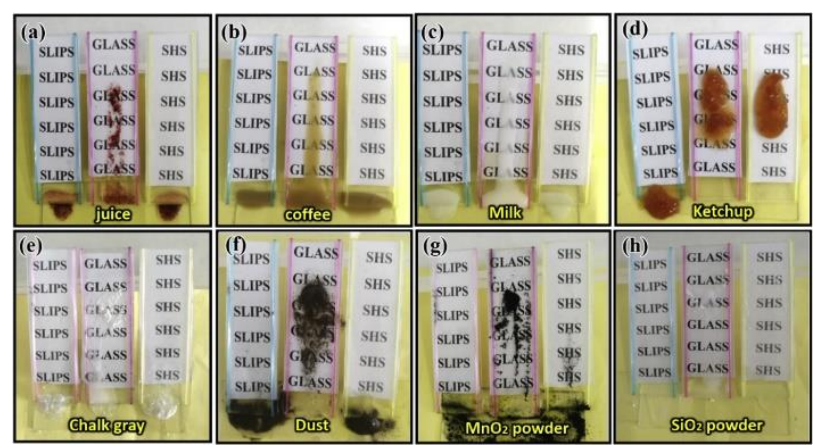

Fig. 28. Anti-fouling and self-cleaning properties of the slippery lubricant-infused surface. Images reported from [54].

SLIPS shows the potential to prevent a wide range of fouling. Zhang et al. reported a mechanical robust SLIPS with a transmittance of $92.01 \%$ were prepared by infusing lubricant oil into PHTSs which were synthesized by combining the hydrothermal and electrospinning methods [54]. Fig. 28 shows the omniphobic nature of SLIPS that can help to protect the surfaces from a wide range of contamination by allowing anti-fouling and self-cleaning using a broad of fluids to remove the particles from the surfaces.

\section{Conclusion}

In this review, we have broadly discussed surfaces in natural or bio-inspired with efficient anti-fouling properties.

Fouling, defined as the undesired adhesion or adsorption of contaminants to a given surface, has various types. Each type of fouling is problematic or dangerous for multiple applications and industrial processes. This review uses a mean-shift method to classify fouling into four main groups, protein fouling, bio- and dust-fouling, macro-inorganic fouling, and micro-inorganic fouling.

Nature's flora and fauna demonstrate a multitude of antifouling lessons that can be mimicked for engineering purposes. Nature frequently uses a combination of methods to successfully prevent fouling. Many of nature's antifouling mechanisms have been studied to unlock their secrets, including examples such as self-cleaning lotus leaf, shark skin, and pitcher plant. These mechanisms include physical and chemical controls such as low drag, low adhesion, wettability, micro-texture, grooming, sloughing, various miscellaneous behaviours, and chemical secretions.

Bio-inspired artificial surfaces imitate the natural strategies in various ways, including synthetic, microstructure, and other strategies. The bio-inspired strategies show great fouling resistance character along with the origin nature surfaces. Moreover, some strategies used to tackle one specific fouling have found unexpected anti-fouling properties in other kinds of fouling which the nature prototype doesn't experience. For example, the SLIPS which shows a good anti-ice-fouling property is inspired by Nepenthes, a plant that grows in ice-free areas, the micro-hoodoo structure which shows a great anti-oilfouling property is inspired by springtail, a tiny insect with no chance to meet oil.

In the real world, the need for the surface is correlated to the working environments, no nature surface is evolved to prevent all types of fouling. The artificial surfaces, however, could combine different features which resist different types of fouling. But at present, no research has reported a surface that shows omni-fouling preventing property. Our review shows that with the combination of synthetic and micro-structure strategies, a surface could show great anti-fouling properties in more than one group like bio- and dust-fouling, and macro-inorganic fouling. Moreover, the SLIPS shows resistant behaviour to various groups of fouling as well. These reports show that an omni-fouling-prevent surface is foreseeable in the future, using mixed strategies and advanced materials. 


\section{References}

1. J.L. Laforte, M.A. Allaire, J. Laflamme, Atmos. Res. 46,143-158 (1998)

2. G. Poots, R.W. Gent, N.P. Dart, J.T. Cansdale, Phil. Trans. R. Soc. A, 358, 2873-2911 (2000)

3. K.J. Leontaritis, G.Ali Mansoori, J. Pet. Sci. Eng. 1, 229-239 (1988)

4. K. Pourabdollah, J. Environ. Chem. Eng. 9, 104552 (2021)

5. S.L. Percival, L. Suleman, C. Vuotto, G. Donelli, Journal of Medical Microbiology, 64, 323-334 (2015)

6. M.P. Schultz, J.A. Bendick, E.R. Holm, W.M. Hertel. Biofouling, 27, 87-98 (2011)

7. Gordon JE. The new science of strong materials, or why you don't fall through the floor. 2nd ed. (Pelican-Penguin, London, UK, (1976))

8. B. Bhushan, Phil. Trans. R. Soc. A, 367, 1445-1486, (2009).

9. S. Zheng, C. Li, Q. Fu, W. Hu, T. Xiang, Q. Wang, M. Du, X. Liu, Z. Chen, Mater. Des. 93, 12 (2015).

10. G.D. Bixler, B. Bhushan, Phil. Trans. R. Soc. A, 370, 2381-2417, (2012).

11. A.K. Halvey, B. Macdonald, A. Dhyani, A. Tuteja, Phil. Trans. R. Soc. A. 377, 20180266, (2019).

12. G. Heydari, E. Thormann, M. Järn, E. Tyrode, P.M. Claesson, J. Phys. Chem. 117, 21752-21762, (2013)

13. X. Wang, H. Liu, W.L. Ma. IFAC-PapersOnLine, 51 ,292-296, (2018).

14. J. Yang, S. Rahardja, P. Fränti, Pattern Recognit. 115, 107874, (2021).

15. G.D. Bixler, A. Theiss, B. Bhushan, S.C. Lee, J. Colloid Interface Sci. 419,114-133, (2014)

16. Q. Yu, Y. Zhang, H. Wang, J. Brash, H. Chen, Acta Biomater. 7, 1550-1557, (2011).

17. M.F.B. Sousa, H.C. Loureiro, C.A. Bertran, Surf. Coat. Technol. 382, 125160, (2020)

18. Z. Guo, W. Liu, Plant Sci. 172, 1103-1112, (2007).

19. K. Rykaczewski, S. Anand, S.B. Subramanyam, K.K. Varanasi, Langmuir, 29, 5230-5238, (2013)

20. T. Sun, L. Feng, X. Gao, L. Jiang, Accounts Chem. Res. 38, 644-652, (2005)

21. X. Chen, R. Ma, H. Zhou, X. Zhou, L. Che, S. Yao, Z. Wang. Sci. Rep. 3, 2515, (2013)

22. S.M. Olsen, L.T. Pedersen, M.H. Laursen, S. Kiil, K.D. Johansen, Biofouling, 23, 369-383, (2007)

23. Z. He, C. Wu, M. Hua, S. Wu, D. Wu, X. Zhu, J. Wang, X. He, Matter, 2, 723-734, (2020)

24. L. Ma, Z. Zhang, L. Gao, Y. Liu, H. Hu, Renew. Energy, 162, 2344-2360, (2020).

25. W. Choi, A. Tuteja, J.M. Mabry, R.E. Cohen, G.H. McKinley, J. Colloid Interface Sci. 339, 208-216, (2009)

26. D. Hou, K.S.S. Christie, K. Wang, M. Tang, D. Wang, J. Wang, J. Membr. Sci. 599, 117708, (2020)

27. W.C. Sherbrooke, A.J. Scardino, R. de Nys, L. Schwarzkopf, Zoomorphology, 126,89-102, (2007).

28. P. Zhang, L. Lin, D. Zang, X. Guo, M. Liu, Small, 13,1503334, (2017)

29. W. Barthlott, C. Neinhuis, Planta, 202,1-8, (1997)
30. R. Jiang, L. Hao, L. Song, L. Tian, Y. Fan, J. Zhao, C. Liu, W. Ming, L. Ren, Chem. Eng. J. 398,125609, (2020).

31. G.D. Bixler, B. Bhushan, Adv. Funct. Mater. 23,4507-4528, (2013)

32. G.D. Bixler, B. Bhushan, J. Colloid Interface Sci. 393,384-396, (2013).

33. Y. Lin, Y. Ting, B. Chen, Y. Cheng, T. Liu. Surf. Coat. Technol. 391, 125663, (2020)

34. D. Brian, B. Bharat, Phil. Trans. R. Soc. A. 368 , 4775-4806, (2010)

35. M. Liu, S. Wang, Z. Wei, Y. Song, L. Jiang, Adv. Mater. 21,665-669, (2009).

36. M.L. Carman, T.G. Estes, A.W. Feinberg, J.F. Schumacher, W. Wilkerson, L.H. Wilson, M.E. Callow, J.A. Callow, A.B. Brennan, Biofouling, 22,11-21, (2006)

37. H. Chien, X. Chen, W. Tsai, Mater. Lett. $\mathbf{2 8 5}, 129098$, (2021)

38. G.D. Bixler, B. Bhushan. Soft Matter, 8, 1127111284 (2012)

39. Y. Fang, G. Sun, Y. Bi, H. Zhi, Sci. Bull. 60,256263, (2015)

40. Y. Yeong, M.C. Gupta, Surf. Coat. Technol. 313,1723, (2017)

41. R. Hensel, A. Finn, R. Helbig, H. Braun, C. Neinhuis, W. Fischer, C. Werner, Adv. Mater. 26, 2029-2033, (2014).

42. K.J. Lu, Y. Chen, T. Chung, Water Res. 162,64-77, (2019).

43. R. Hensel, C. Neinhuis, C. Werner, Chem. Soc. Rev.45,323-341, (2016).

44. T.T.Y. Tan, M.R. Reithofer, E.Y. Chen, A.G. Menon, T.S. Andy Hor, J. Xu, J. Min Chin, J. Am. Chem. Soc. 135 ,16272-16275, (2013).

45. K. Karnjana, C. Soowannayan, K. Wongprasert. Fish Shellfish Immunol. 88, 91-101, (2019)

46. T. Suvega, K. Arunkumar. Biocatalysis and Agricultural Biotechnology, 19,101136, (2019)

47. G. Bonthond, T. Bayer, S. A. Krueger-Hadfield, N. Strck, F. Weinberger. The ISME Journal, (2021).

48. C. Baum, F. Simon, W. Meyer, L. Fleischer, D. Siebers, J. Kacza, J. Seeger, Biofouling, 19,181-186, (2003)

49. C. Baum, W. Meyer, R. Stelzer, L.-G. Fleischer, D. Siebers, Mar. Biol. 140, 653-657, (2002)

50. K. Liu, C. Wang, J. Ma, G. Shi, J. Wang, Proc. Natl. Acad. Sci. 113, 14379, (2016)

51. E. V. Gorb, M. J. Baum, S. N. Gorb, Sci. Rep. 3,3078, (2013)

52. D. Labonte, A. Robinson, U. Bauer, W. Federle, Acta Biomater. 119,225-233, (2021).

53. T. Ko, S. Cho, S. Kim, Y.A. Lee, D.H. Kim, W. Jo, H. Kim, S. Yang, K.H. Oh, M. Moon, J. Hazard. Mater. 410, 124549, (2020).

54. M. Zhang, J. Yu, R. Chen, Q. Liu, J. Liu, D. Song, P. Liu, L. Gao, Jun Wang, J. Alloys. Compd. 803,51-60, (2019)

55. H. Zhang, Y. Liang, P. Wang, D. Zhang, Prog. Org. Coat. 132,132-138, (2019)

56. P. Wang, D. Zhang, Z. Lu, Colloids Surf. B Biointerfaces, 136,240-247, (2015). 
57. M. Xie, Y. Wang, W. Zhao, Colloids Surf. B Biointerfaces, 197,111375, (2021) 